\title{
Environmental fluctuations and asymmetrical dispersal: generalized stability theory for studying metapopulation persistence and marine protected areas
}

\author{
Christopher M. Aiken*, Sergio A. Navarrete \\ Estación Costera de Investigaciones Marinas, Center for Advanced Studies in Ecology and Biodiversity, \\ and Laboratorio Internacional de Cambio Global (LINC, CSIC-PUC), Pontificia Universidad Católica de Chile, \\ Casilla 114-D, Santiago, Chile
}

\begin{abstract}
Dispersal of individuals among subpopulations is a key process underlying metapopulation dynamics. Many metapopulation models, including those of coastal benthic organisms under marine reserve scenarios, have assumed a particular and time-independent dispersal pattern. The behavior of such models, however, may be sensitive to more realistic representations of oceanic dispersal. We examine the importance of environmental variability and dispersal characters for metapopulation persistence using a space-limited metapopulation model, in which the dispersal phase is represented by a connectivity matrix and environmental fluctuations by stochastic perturbations of adult abundances. The model is suited to marine organisms, but the same principles apply to other systems. When dispersal is asymmetrical, as expected in the presence of a dominant current, environmental variability can allow the metapopulation to persist, even when the per capita larval production rate is too low to otherwise sustain the population. This suggests that metapopulations inhabiting finite ranges in an advective environment may be more susceptible to variations related to climate change. Generalized stability theory is a powerful tool for identifying the local populations that have greatest impact on the metapopulation, and hence the optimal sites for protection from exploitation. We show that the inclusion of realistic environmental variability and complex dispersal patterns in models of marine reserve networks can bring unsuspected and sometimes largely positive effects for conservation and management of benthic species. Thus, marine reserve monitoring of abundance and recruitment in systems with longshore currents should include the region of longdistance dispersal of the species.
\end{abstract}

KEY WORDS: Marine reserves - Marine protected area · Larval dispersal · Management • Population dynamics $\cdot$ Spatially explicit models $\cdot$ Generalized stability $\cdot$ Reactivity

Resale or republication not permitted without written consent of the publisher

\section{INTRODUCTION}

Since the introduction of the first metapopulation models several decades ago (Levins 1969), a large number of theoretical models, using a wide range of approaches, have illustrated the consequences that spatial structure and dispersal can have on the dynamics and persistence of populations and on their interactions with other species (see Taylor 1990, Hanski \& Gilpin 1991, Hanski \& Simberloff 1997, Sale et al. 2006,
Gaines et al. 2007 and references therein). But despite its importance, the effect on model outcomes of assumptions underlying the way organisms disperse across space is not fully understood (Speirs \& Gurney 2001, Chesson \& Lee 2005, Anderson et al. 2008). Because dispersal kernels, which define the probability distribution of recruitment as a function of release and settlement location (e.g. Chesson \& Lee 2005), are usually poorly resolved empirically, the majority of theoretical models have explicitly or implicitly used either 
uniform, Gaussian, or leptokurtic shapes for dispersal. While these studies most commonly consider situations of strong self-recruitment, or even symmetrical dispersal, in many real systems individuals are found in an advective environment that generates asymmetrical dispersal in which self-recruitment may be very weak (Speirs \& Gurney 2001, Anderson et al. 2008). Such is the case of marine coastal organisms dispersing in a dynamic ocean, where dispersal across the metapopulation can be highly asymmetrical (Largier 2003, Byers \& Pringle 2006, Kaplan 2006, Aiken et al. 2007). In strongly advective environments, the persistence of a population can be strongly dependent upon details of the kernel shape and of the level of environmental variability (Speirs \& Gurney 2001, Pachepsky et al. 2005, Byers \& Pringle 2006, Anderson et al. 2008, Lutscher et al. 2010). In the following, we demonstrate that environmental variability can have important consequences for population persistence in a geographically finite habitat with net advection.

The majority of marine organisms, including fish and invertebrates, possess a planktonic phase that can be transported in the ocean over periods from hours to months while they develop into competent larvae that can then settle in the adult habitat. Since the adult stages of many of these species are sedentary, the pelagic dispersal phase allows the species to colonize and invade new habitat away from the parental population, and represents the main vehicle for genetic interchange among existing local populations (Gaines \& Bertness 1992, Palumbi 1994, Largier 2003, Siegel et al. 2008). In most cases, the horizontal swimming speeds of larvae are not sufficient to overcome common ocean current velocities, and their dispersal is therefore highly dependent on ocean currents. The fact that these ocean currents are intrinsically variable across a range of spatial and temporal scales by itself introduces a high level of variability into recruitment.

Since benthic marine species are commercially exploited and/or are in need of conservation measures to prevent local or global extinction (Botsford et al. 1997, Jackson et al. 2001), much of the recent modeling of marine metapopulations has been motivated by the need to predict the benefits of marine protected areas (MPAs) as tools for conservation and sustainable management (e.g. Hastings \& Botsford 1999, 2006, Botsford et al. 2001, Kaplan \& Botsford 2005, Kaplan 2006). Given that marine networks should be self-sustaining and net exporters of young to unprotected populations, the details of larval dispersal are fundamental to effective marine reserve design. As per metapopulation models for terrestrial organisms, dispersal is typically modeled using simple or complex hypothetical dispersal kernels (Hastings \& Botsford 1999, Botsford et al. 2001, Lipcius et al. 2001, Lockwood et al. 2002, Kaplan
2006). Analysis of such idealized metapopulation models has been used to investigate necessary conditions for metapopulation persistence at equilibrium, and marine reserve size and design that maximize fisheries yield. However, there is a growing recognition of the need to use more realistic kernels that include the intrinsic time dependence of larval production and oceanic dispersal (Aiken et al. 2007, Edwards et al. 2007, Paris et al. 2007, Siegel et al. 2008). In addition, in many cases long-term solutions are not the only time scales of interest, i.e. the temporal dynamics of the population before reaching such equilibrium might provide answers to many ecological and management issues (Hastings \& Higgins 1994).

The response of a dynamical system to external perturbation is composed of transient (short time) and asymptotic (long time) solutions. Traditional stability analysis concentrates on the asymptotic response, i.e. stability and resilience, but recent decades have brought an increasing awareness of the potential importance of the transient phase (Hastings 2004). In the ecological context, Neubert \& Caswell (1997) demonstrated the possibility that perturbations may grow rapidly for significant periods of time despite the fact that all disturbances decay in the asymptotic limit. This phenomenon, referred to as reactivity, has been shown to play a vital role in the generation of Turing instabilities (Neubert et al. 2002). Hastings \& Higgins (1994) showed that such transient phenomena are particularly common in spatially structured models, such as populations of sessile species connected by dispersal. Recently, Anderson et al. (2008) applied concepts of reactivity to populations that inhabit an advective environment, determining the spatial wavelengths that create the greatest downstream amplification of an initial disturbance to the population. Their work concentrated upon the transient behavior of individual perturbations to ecological systems. Here we extend this work to investigate the sensitivity of the dynamics of marine populations to natural continuous variability in different dispersive environments, using a simple and commonly-used space-limited model of species abundance. We demonstrate that the existence of a net mean a longshore displacement of larvae in a finite habitat renders the system reactive and can fundamentally affect metapopulation response to environmental variability. In addition, we present a generalized means for determining the spatial structure that produces the greatest transient growth, which can be applied in realistic ocean models to determine the 'optimal' position of an MPA. Such information on metapopulation sensitivity is also of importance for understanding possible metapopulation response under altered environmental conditions (e.g. Snyder 2009), such as those due to climate change. Although 
our model is targeted at marine species, it is sufficiently simple and general to be relevant to a wide variety of systems whose population dynamics depend on connectivity.

\section{GENERALIZED STABILITY OF A SPACE-LIMITED METAPOPULATION}

\section{Metapopulation model}

We employed a simple metapopulation model based on that of Roughgarden et al. (1985) but where spatial patterns of larval exchange are explicitly included via a connectivity matrix. The model can be written as:

$$
\mathrm{d} \mathbf{n} / \mathrm{d} t=(f \mathbf{S}(\mathbf{n}) \mathbf{C}-\mathbf{M}) \mathbf{n}
$$

where $\mathbf{n}$ is the state vector containing the number of individuals in each local population; $\mathbf{C}$ is the connectivity matrix, which defines the probability of competent larval delivery to each of the $J$ local populations at time $t$, such that the diagonal of $\mathbf{C}$ determines the level of self-recruitment; $f$ is the fertility, or net larval production rate per adult per unit time, subsuming the larval mortality term; $\mathbf{S}(\mathbf{n})$ is the density-dependent settlement term, which in this case increases linearly with the fractional availability of free substrate and may be cast in matrix form as:

$$
\mathbf{S}(\mathbf{n})=\mathbf{I}-\mathbf{\Sigma}(\mathbf{n})
$$

where $\mathbf{I}$ is the identity matrix. $\boldsymbol{\Sigma}(\mathbf{n})=\operatorname{diag}\left(\mathrm{n}_{1} / \mathrm{N}_{1}, \mathrm{n}_{2} / \mathrm{N}_{2}\right.$, $\left.\ldots \mathrm{n}_{J} / \mathrm{N}_{J}\right), \operatorname{diag}()$ denotes the matrix with the elements indicated along the diagonal and zeros elsewhere, and $\mathrm{N}_{j}$ is the limiting abundance in population $j$, which is determined by a fixed availability of free space in that population and the per capita use of this resource in that population. For simplicity, we assume a homogeneous availability of space, that is $\mathbf{N}_{j}=\mathrm{N}$. Lastly, $\mathbf{M}$ is the mortality rate matrix $\operatorname{diag}\left(m_{1}, m_{2}, \ldots m_{J}\right)$, where the $m_{j}$ are the local mortality rates per unit time.

The density dependence term (Eq. 2) renders the model (Eq. 1) non-linear. However, if a solution to (Eq. 1) is given by $\mathbf{n}=\mathbf{n}^{*}$, then perturbations $\mathbf{v}=\mathbf{n}-\mathbf{n}^{*}$ to this state may be described to order $\left(\|\boldsymbol{v}\|^{2} / \mathrm{N}\right)$ by the tangent linear model (TLM)

$$
\mathrm{d} \mathbf{v} / \mathrm{d} t=\left(f \mathbf{S}\left(\mathbf{n}^{*}\right) \mathbf{C}-\mathbf{M}\right) \mathbf{v}-f \boldsymbol{\Sigma}(\boldsymbol{v}) \mathbf{C} \mathbf{n}^{*}=\mathbf{A} \mathbf{v}
$$

This approximation (3) remains valid as long as $\|\boldsymbol{v}\| \ll \mathrm{N}$.

A is referred to as the tangent linear operator of the non-linear model (Eq. 1). The dynamics of population growth are contained within the TLM; only the magnitude of the density-dependence term varies between linear and non-linear models. In general, Eq. (3) predicts the trend of population growth or decay in Eq. (1), but overestimates the magnitude. Thus, when densitydependent settlement effects are small, the 2 systems agree closely.

\section{Generalized stability theory}

The traditional means to assess the stability of a dynamical system such as Eq. (1) is to identify the fixed equilibrium points and then perform an eigenvector decomposition (or Lyapunov analysis for time-dependent, or 'non-autonomous,' systems) of the model linearized about those steady states (Lewontin 1969). Central to this approach is the assumption that the system will reside close to a fixed point provided that all small perturbations to the system decay. The population abundance in the long-time limit is determined by the largest eigenvalue or Lyapunov exponent $\lambda$. The value of $-\operatorname{Re}\{\lambda\}$ is referred to as the resilience (Neubert $\&$ Caswell 1997), as it gives the speed at which the disturbed population returns to its original equilibrium abundance. A resilience that is negative implies unbounded exponential growth of any small initial disturbance and hence instability of the fixed point, while positive resilience ensures that all perturbations to the fixed point will decay in the long-time (asymptotic) limit and hence that the system is stable. By focusing on the concept of resilience and asymptotic solutions, early work on metapopulation stability implicitly assumed that growth over short time scales (transient growth) either could not occur or was not important. In recent decades, however, it has been recognized that transient behavior can in fact dominate population dynamics in real systems (Hastings 2004), and can occur even when the resilience is positive (Neubert \& Caswell 1997).

In order for a metapopulation described by Eq. (1) to survive in the long term, it is sufficient that the tangent linear operator linearized at $\mathbf{n}=0$ (referred to here as $\mathbf{A}_{0}$ ) possesses at least 1 positive eigenvalue. A positive eigenvalue of $\mathbf{A}_{0}$ means that a small number of individuals added to an initially empty habitat will grow exponentially and, therefore, population survival in the asymptotic limit is guaranteed. This is equivalent to the invasability criterion (e.g. Chesson 2003). The densitydependent term $\mathbf{S}(\mathbf{n})$ in the non-linear model (1) prevents unbounded exponential growth, and any initial population monotonically approaches an equilibrium state. For each choice of dispersal kernel, a critical value of the net larval production rate, $f=f_{\mathrm{C}}$, may be determined at which the first positive eigenvalue of $\mathbf{A}_{0}$ appears, and hence where the resilience first becomes negative.

Although $f>f_{\mathrm{c}}$ guarantees that the metapopulation will persist in the long term, $f<f_{\mathrm{c}}$ does not necessarily 
mean that all disturbances must decay monotonically. Under certain quite general circumstances, it is possible for large and sustained transient growth of perturbations to occur, prior to their eventual decay. Systems that possess this property are referred to as 'reactive' (Neubert \& Caswell 1997). A prerequisite for reactivity is that the eigenvectors of $\mathbf{A}$ are non-orthogonal, in which case the system is referred to as non-normal (Trefethen et al. 1993, Farrell \& Ioannou 1996). Note, however, that not all non-normal matrices are necessarily reactive (Neubert \& Caswell 1997). The way in which the non-normalilty of a system permits the growth of disturbances over short time scales can be most easily understood geometrically. Fig. 1 illustrates the evolution of a perturbation composed of a sum of 2 non-orthogonal eigenvectors. Although both eigenvectors decay in time, their sum increases in length over the same time period. That is, a sum of nonorthogonal eigenvectors can grow over a transient period even though each eigenvector decays monotonically. The transient perturbation growth that occurs in higher dimensional reactive systems occurs through this same process. It may be appreciated that the potential for transient growth increases together with the non-orthogonality between the eigenvectors, and hence the reactivity is tightly bound to the degree of non-normality.

Although a transiently growing perturbation must eventually decay, the transient behavior can last many generations before the system reaches asymptotic behavior (Hastings \& Higgins 1994). Moreover, real ecological systems are constantly subject to perturbations in the form of environmental fluctuations, and as a result the transient growth phase can become important in determining the long-time mean state of the system. In fact, when a dynamical system is reactive, it may sustain a high degree of variability when exposed to stochastic forcing, despite being asymptotically stable in the traditional eigenvalue sense (e.g. Aiken et al. 2003). The transient behavior of small perturbations is
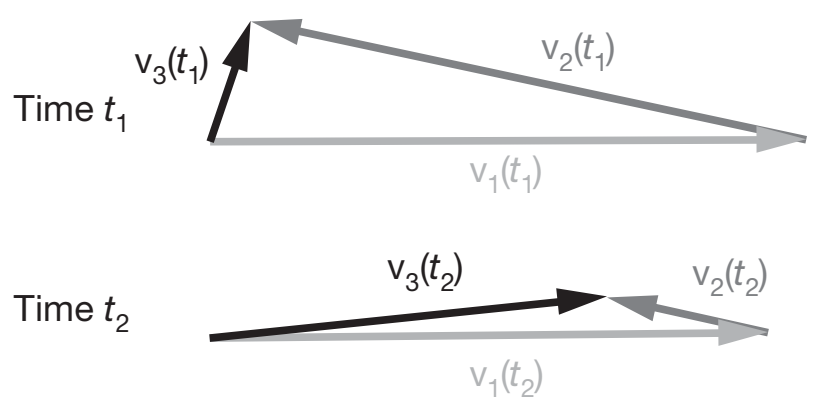

Fig. 1. Geometrical demonstration of how the sum $\left(v_{3}\right)$ of 2 non-orthogonal vectors $\left(\mathrm{v}_{1}\right.$ and $\left.\mathrm{v}_{2}\right)$ can grow in length for the transient time period from $t_{1}$ to $t_{2}$, even while each of the component vectors decreases in length over the same time period commonly the most relevant dynamic in many ecological problems (Neubert \& Caswell 1997, Hastings 2004, Anderson et al. 2008).

In general, the non-normality (and hence reactivity) of a system is related to the asymmetry of the underlying dynamical operator. In the spatially structured metapopulation model considered here, it is the asymmetry of dispersal that renders the system non-normal or reactive and hence allows the transient phase to become important. As a result, in ecological systems with net advection and subject to continual environmental forcing, it is the so-called 'generalized stability theory', which considers the role of transient perturbations, that represents the more appropriate analysis for understanding system behavior within time scales relevant to many ecological, conservation, and management problems.

Whereas traditional stability analysis involves an eigenvalue decomposition of the dynamical operator A, the analysis of the transient phase in generalized stability theory proceeds with a singular value decomposition (SVD) of the 'propagator' $\mathbf{R}(t)=\mathrm{e}^{\mathbf{A} t}$. (Details of the SVD are given in Appendix 1.) The SVD yields not only the maximum growth rates over time $t$ (the singular values), but also the spatial structure of the perturbations that give rise to this maximum or optimal growth (the singular vectors). In this way, the singular vectors indicate which local populations are most sensitive to variations in their adult abundance. For each time $t$, the singular vector (initial condition) associated with the largest singular value (amplification factor $\rho$ ) is commonly referred to as the 'optimal perturbation' for that time span, and that for all times as the 'global optimal'. We show below that relatively large and long-lived perturbations lasting many thousands of generations can occur in the simple metapopulation model (Eq. 1) even when no growth is possible in the asymptotic limit.

The reactivity, given by the largest eigenvalue of the real symmetrical matrix $\left(\mathbf{A}+\mathbf{A}^{+}\right) / 2$ (Neubert \& Caswell 1997), represents the maximum instantaneous perturbation growth rate and can be shown to be identical to the leading singular value of $\mathbf{R}(t)$ as $t$ approaches 0 (Farrell \& Ioannou 1996). The 'amplification envelope' of Neubert \& Caswell (1997) is identically the value of the leading singular value as a function of $t$. In fact, the matrix 2-norm used to determine the maximum growth rates requires a partial SVD of $\mathbf{R}(t)$. By performing a full SVD, and not just calculating the leading singular value to determine the norm, the system sensitivity to spatial forcing patterns can be determined completely. It will be shown below that the singular vectors provide important information for understanding the response of a metapopulation to spatially structured disturbances, and hence for marine reserve design. 


\section{Optimal perturbations in symmetrical and asymmetrical dispersal environments}

The generalized stability analysis outlined above was applied to the TLM (Eq. 4) (the linearized version of the single species metapopulation model, Eq. 1) under a set of varying dispersal scenarios. The modeled metapopulation was considered to be composed of $J=10$ equally spaced local populations inhabiting a finite section of coast. The results that follow are insensitive to the exact value of $J$. The time-independent dispersal kernel used for constructing the connectivity matrix $\mathbf{C}$ was Gaussian, as would occur for an ocean circulation whose mean and variance are stationary and homogeneous. Thus, the connectivity matrix is completely defined by a mean $\mu$ and standard deviation $\sigma$. In order to illustrate the effect of increasing dispersal asymmetry, and hence increasing system reactivity or non-normality, $\mu$ took values between 0 and 10 , while $\sigma$ was kept fixed at 3 units of alongshore separation of local populations. (Given the correspondence between dispersal asymmetry and non-normality, our focus is upon $\mu$. The effect of changing $\sigma$ is discussed below.) Increasing the mean dispersal distance can be thought of as either increasing the mean alongshore oceanic transport or increasing the larval pelagic development time (PLD). Connectivity matrices with $\mu=0$ show highest settlement along the diagonal ('selfrecruitment'), while for $\mu=5$, highest settlement occurs downstream from the releasing population and levels of self-recruitment are low (Fig. 2). A constant metapopulation mortality rate of $0.01 \mathrm{wk}^{-1}$ and maximum population size of 100 individuals were used in the model. These values are nominal and the results that follow are not sensitive to their choice. Sensitivity to the choice of $\mu$ and $\sigma$ are discussed below.

Environmental variability was included in the model through the random addition and removal of individuals from each population, represented in the model through the addition of a stochastic forcing term $\xi(t)$ to the state vector $\mathbf{n}$, viz.

$$
\mathbf{n}(t+\delta t)=\mathbf{n}(t)+\mathbf{A n}(t)+\xi(t) \delta t^{1 / 2}
$$

where each element $\xi_{i}$ of $\xi(t)$ was an independent normally-distributed random variable with $\left\langle\boldsymbol{\xi}_{i}(t)\right\rangle=0$ and $\left\langle\xi_{i}(t)^{2}\right\rangle=0.01$, where \langle\rangle represents the value averaged over time $t$. Thus, the stochastic forcing represented no net contribution of individuals to the metapopulation, and modulation of the population abundances was trivially small. Note that the multiplication by $\delta t^{1 / 2}$ instead of $\delta t$ in (Eq. 4) is necessary to preserve the variance properties of the time-integrated white noise (Weiner) process (Gardiner 1985). In order to ensure that the solution remained within the physical limits, the condition

$$
0 \leq \mathrm{n}_{j} \leq \mathrm{N}
$$

was enforced at each time step.

\section{RESULTS}

\section{Idealised model}

The effect of increasing alongshore flow, and hence dispersal asymmetry, upon metapopulation sensitivity is illustrated in Fig. 3. The minimum larval production rate required for metapopulation persistence $f_{\mathrm{C}}$ increases with increasing dispersal asymmetry $\mu$ (Fig. 3a) due to the ever greater advective loss of larvae from the metapopulation. As indicated by the value of the largest singular value, the potential magnitude of sub-critical $\left(f<f_{\mathrm{c}}\right.$ ) perturbation growth also increases with $\mu$, while for $\mu=0$, all perturbations to the metapopulation decay on all time scales, and transient growth by a factor of approximately 300 is possible at $\mu=4$ and almost 2000 when $\mu=5$ (Fig. 3b). The time taken for maximum growth to occur (not shown) also increases with $\mu$, reaching 67 wk when $\mu=5$. The form
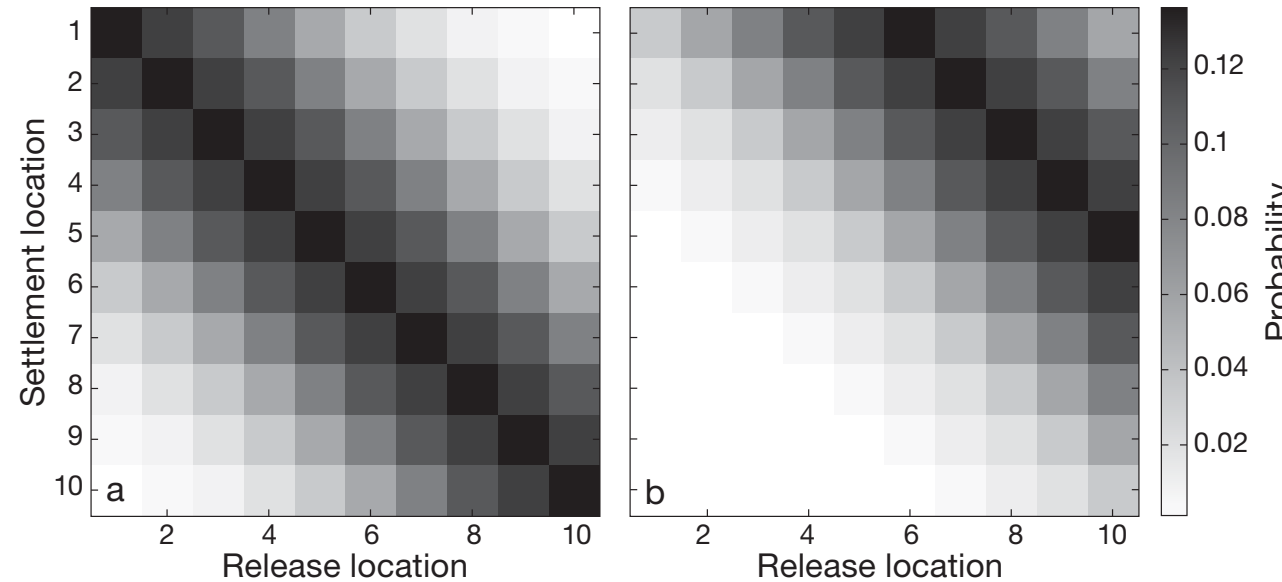

Fig. 2. Connectivity matrices for (a) $\mu=0$ and (b) $\mu=5$. Grayscale indicates the probability that a larva released at a given location along the horizontal axis settles at a given location on the vertical axis 
of the least damped eigenvector (i.e. the spatial perturbation structure that dominates in the asymptotic limit; Fig. 3c) and global optimal calculated from an SVD (the spatial perturbation structure that grows the most over any time scale; Fig. 3d) also change with increasing $\mu$. The asymmetry in the least damped eigenvector increases with $\mu$, increasingly weighted to the downstream end of the model habitat. This is opposite to the change in the structure of the global optimal, which is weighted increasingly upstream. In all cases, however, the global optimals evolve over time to take the form of the least damped eigenvector (Fig. 4a,b). Thus, the addition of just 1 individual to the upstream population in the model with $\mu=5$ can cause the net metapopulation abundance to increase by 2000 individuals. The perturbation grows over the course of a year and lasts for a period of many thousands of generations before finally decaying.

The conclusions of the analysis presented above regarding the possibility for perturbation growth in the linearized model carry over to the full non-linear model (Eq. 1). To demonstrate this, the non-linear model was numerically integrated forward in time using a 4thorder Runge-Kutte algorithm for the cases of $\mu=0$ and $\mu=5$. Using a super-critical fertility rate $\left(f>f_{\mathrm{c}}\right)$ and nonzero initial conditions, in each case the metapopulation approaches a steady non-zero equilibrium state. The effect of perturbing these steady super-critical systems using the corresponding global optimals is shown in Fig. 4c,d. A large transient population growth occurs in the non-linear model with $\mu=5$ (Fig. $4 \mathrm{~d}$ ), but none occurs in the model with $\mu=0$, consistent with the linear analysis (Fig. 4c).

Although the population growth seen in Fig. $4 \mathrm{~d}$ is transient, and the perturbed metapopulation eventually returns to the equilibrium solution (albeit only after 7 to $20 \mathrm{yr}$ ), the inclusion of the environmental variability term $\xi(t)$ can produce permanent alteration of the mean metapopulation abundances when dispersal is asymmetrical and hence the dynamical operator is non-normal. Fig. 4e,f illustrates the metapopulation trajectories for symmetrical and asymmetrical dispersal from simulations of the stochastically forced model when the values used for $f$ were sub-critical $\left(f<f_{\mathrm{c}}\right)$, such that no population could be sustained without external forcing. The fact that the value of the least damped eigenvalue $\lambda$ is greater for $\mu=0$ than that for $\mu=5$ indicates that exponential decay of perturbations in the long-term limit is more rapid in the latter case. Nevertheless, the addition of stochastic forcing sustains a robust and persistent metapopulation in the system with $\mu=5$, but not when $\mu=0$. In addition, for $\mu=$ 5 , the fluctuations in the local population abundances are of far greater amplitude than that of the forcing.
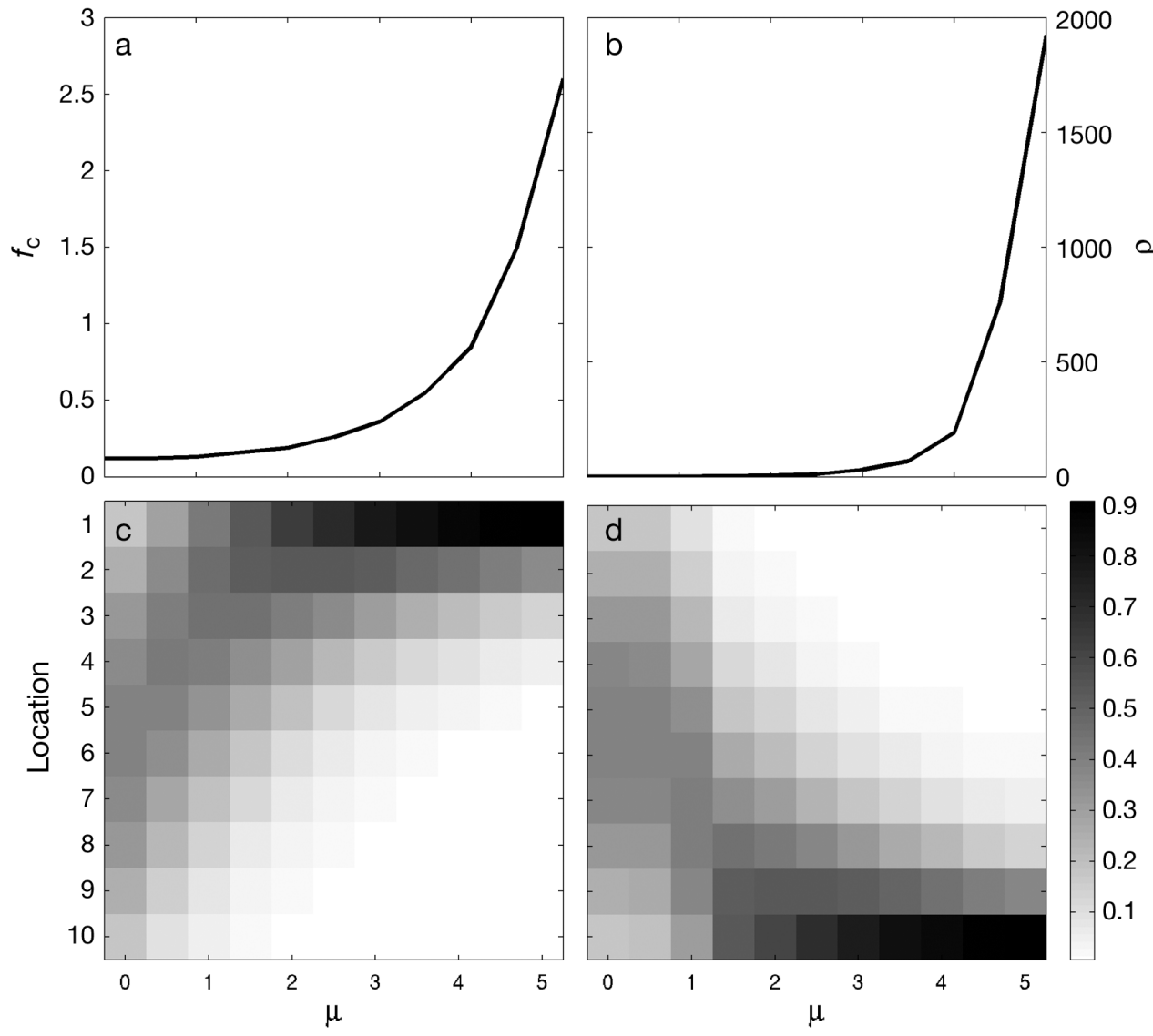

Fig. 3. (a) Critical fertility rate $f_{\mathrm{c}}$ (b) maximum linear growth or amplification factor $\rho$ (largest singular value) for a single disturbance to the linearized system, (c) structure of the least damped eigenvector from traditional stability analysis, and (d) structure of the global optimal perturbation from singular value decomposition analysis (see main text: 'Generalized stability theory'), each as a function of the mean dispersal distance $\mu$. In (c) and (d), location 1 (10) is downstream (upstream) 
The possibility for transient growth depends completely on the fact that the average alongshore dispersal distance $\mu$ is non-zero. When $\mu=0$, the system remains unreactive regardless of the value of $\sigma$. However, when $\mu>0$, the value of $\sigma$ does influence the magnitude of the potential transient growth. In particular, strong transient growth becomes possible when $\mu>\sigma$ (see Fig. 3b), corresponding to the point at which self-recruitment becomes weak.

In addition to increasing the sensitivity to external forcing, non-normality of a dynamical operator increases the sensitivity to changes in the operator itself. Fig. 5 illustrates how the spectrum of eigenvalues of $\mathbf{A}$ varies when the model itself is randomly perturbed. Each small dot of the figure corresponds to the real and imaginary parts of an eigenvalue of the perturbed matrix $\mathbf{A}+\mathbf{E}$, where $\mathbf{E}$ is a random matrix with $\|\mathbf{E}\|<10^{-4}$ for the 2-norm. It can be seen that for $\mu=5$, these small changes to the dynamical operator can have large con- sequences for the eigenspectrum, relative to that which occurs for the case of $\mu=0$. In particular, the operator perturbations commonly increase the value of the least damped eigenvalue, such that the system moves to a state of instability. The random matrices $\mathbf{E}$ represent variations to the model dynamics themselves, such as altered dispersal conditions or mortality rates. Thus, the existence of a mean alongshore flow also has the potential to amplify natural variability in the factors controlling metapopulation dynamics, causing the system to switch between super- and subcritical states.

\section{Realistic example}

The spatial structure of the metapopulation and corresponding connectivity matrices used above, while physically based, are highly idealized. While much
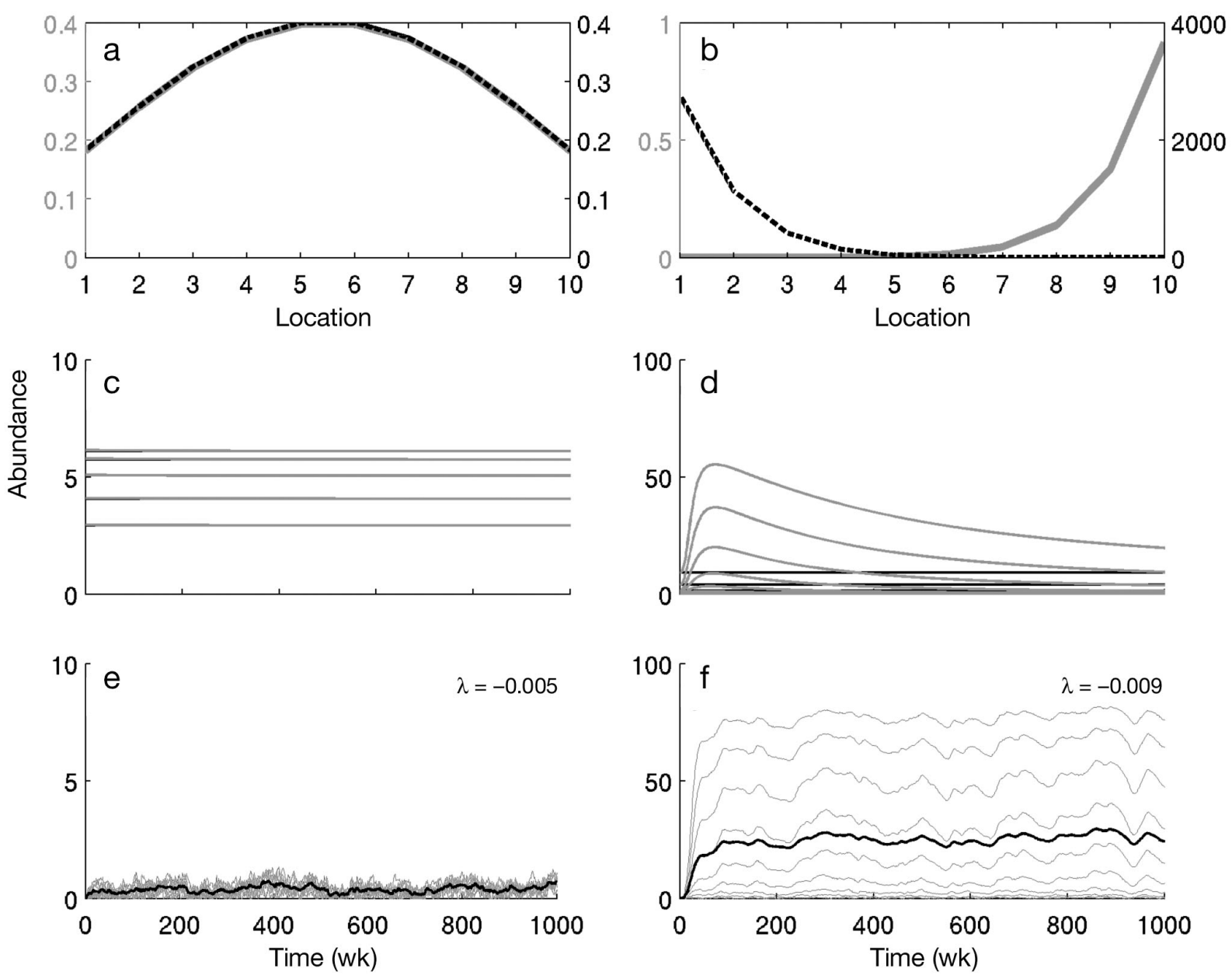

Fig. 4. (a,b) Global optimal perturbation (solid, left axis) and response (dashed, right axis) of the linearized system for the model with (a) $\mu=0$ and (b) $\mu=5$. (c,d) Evolution of equilibrium solution (shown in black) perturbed with optimal perturbation (grey) for (c) $\mu=0$ and (d) $\mu=5$. (e,f) Stochastically (white noise) forced damped (non-self-sustaining) model with (e) $\mu=0$ or (f) $\mu=5$, where the value of $\lambda$ is the largest eigenvalue of the linearized system. (c-f) Each light line represents a population, and the black line is the mean of all populations. Note that in (a) and (c), the black lines are overlapped exactly by the grey lines 
knowledge can be gained from these simple systems both for metapopulation dynamics in general and marine reserve designs in particular (e.g. Botsford et al. 2001, Lockwood et al. 2002, Kaplan 2006), dispersal in real systems is expected to depart significantly from Gaussian and spatial homogeneity (e.g. Aiken et al. 2007). In fact, small-scale features of the coastal geometry and the character of the nearshore circulation (Largier 2003) can produce highly irregular dispersal kernels. Here a connectivity matrix for the central Chilean coast was synthesized using a high-resolution simulation of the local ocean circulation, based on the model described by Aiken et al. (2007, 2008) (Fig. 6a). Trajectories of passive larvae released from 100 locations evenly spaced along the coast were determined by direct integration with the modeled velocity over a 4 yr period. Given the relatively high resolution of the model and its resulting turbulent flow field, no additional diffusivity was added to the particle trajectories. The mean connectivity matrix shown in Fig. 6b was determined by calculating the probabilities of returning to shore after a larval development time of $45 \mathrm{~d}$, typical of many coastal benthic species (O'Connor et al. 2007), as a function of release location. The optimal perturbation to the model using this connectivity matrix is shown in Fig. 6c, together with the model response to this perturbation. It may be seen that, in general, the modeled metapopulation displays greatest sensitivity to changes in the abundances of the populations towards the northern end of the domain. i.e. an increase (decrease) in the abundance in the north, for instance through establishment of a new protected (fishing) area, produces a 4 -fold increase (decrease) in

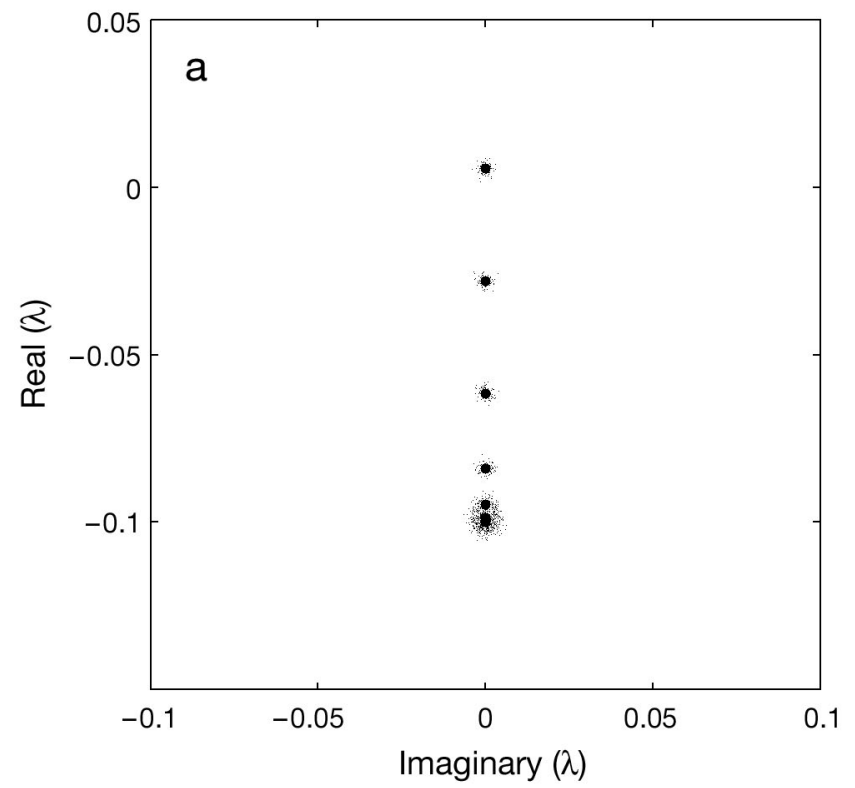

the abundances in the south of the metapopulation approximately 1 yr later. It may be noted, however, that there exists significant alongshore variability in the amplitude of the population response that could not have been guessed. The detail of this variability allows the potentially most important local populations to be identified, and hence may be of vital importance when considering measures of protection for particular coastal areas.

\section{DISCUSSION AND CONCLUSION}

A wide variety of models have shown that dispersal of individuals among local populations and over ecological time scales can have profound consequences on population dynamics, persistence, species interactions, and diversity (e.g. Hanski \& Gilpin 1991, Marquet \& Velasco-Hernández 1997, Pulliam 2000, Snyder \& Chesson 2003, Katul et al. 2005, Sale et al. 2006, Wieters et al. 2008). Yet, we are just beginning to fully appreciate its consequences under realistic scenarios (Roughgarden 2006). In particular, the Gaussian, spatially homogeneous and time-invariant dispersal kernels among local populations used in most models are probably the exception rather than the norm in natural systems (Lockwood et al. 2002, Snyder \& Chesson 2003, Chesson \& Lee 2005, Katul et al. 2005). Although the simple model (Eq. 1) does not include effects such as age structure, adult migration, and seasonal dynamics, given the ubiquity of net longshore transport and high levels of external variability in the coastal ocean (Largier 2003, Byers \& Pringle 2006, Siegel et al. 2008),

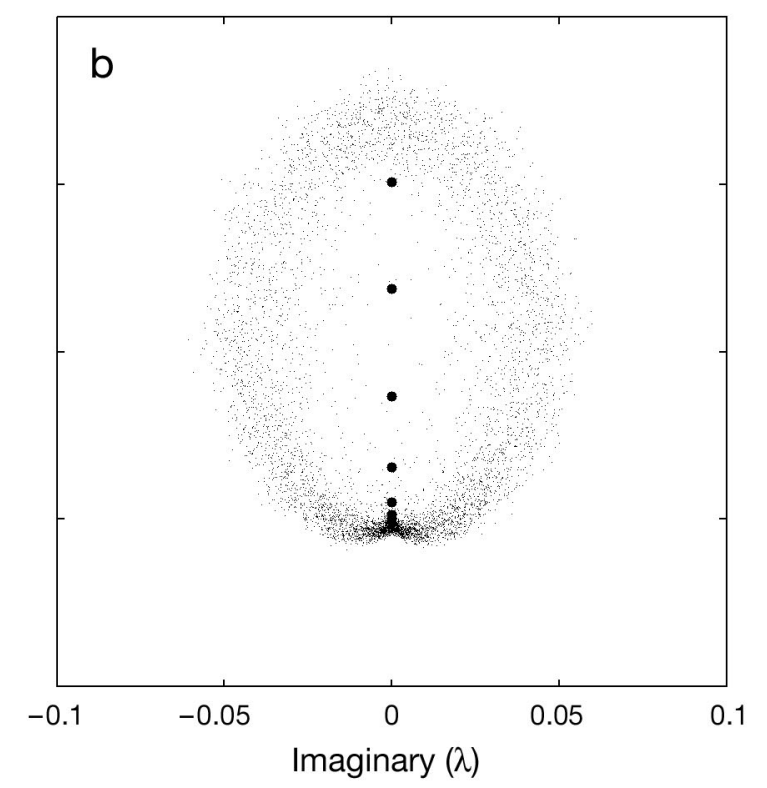

Fig. 5. Spectrum of eigenvalues of the unforced (large dots) and randomly perturbed (small dots) model for (a) $\mu=0$ and (b) $\mu=5$ 

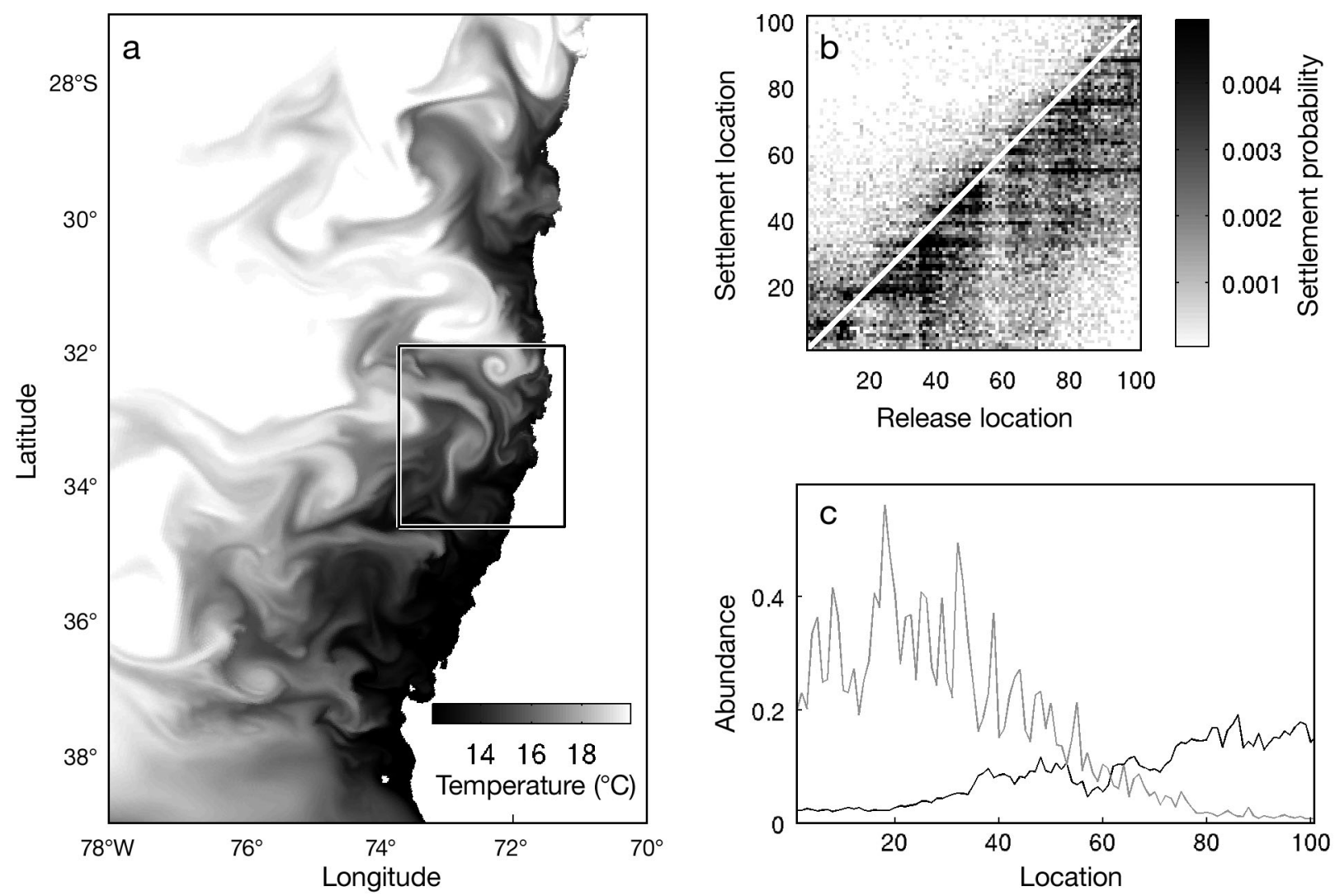

Fig. 6. (a) Snapshot of sea surface temperature from the numerical model of central Chile used to calculate a connectivity matrix for the region marked by the square. (b) Connectivity matrix for a larval dispersal time of $45 \mathrm{~d}$. (c) Global optimal perturbation (black line) and response after 1 yr (grey line) for model with connectivity matrix as shown in (b). Abundance is normalized such that the size of the initial perturbation is 1

the results presented here have relevance for the dynamics of most marine populations and many nonmarine systems with a dispersive life history phase occupying an advective habitat. We submit that the general approach presented here can open new lines of research into the design and evaluation of marine reserve networks, and that the inclusion of complex but more realistic ocean dynamics can bring unsuspected positive conclusions on the requirements for the persistence of exploited or endangered populations.

The importance of examining transient dynamics to address many general ecological and applied questions was well summarized by Hastings (2004), and the potential for such transients in populations of species with space-limited recruitment was demonstrated earlier by Hastings \& Higgins (1994). Here we have shown that the degree of dispersal asymmetry controls the sensitivity of a space-limited metapopulation model to both external stochastic forcing and altered dynamics. When dispersal was symmetrical, the behavior of the model was well predicted by traditional stability analysis, and no complex transient behavior was possible. Under this scenario, whenever the per capita repro- ductive output ( $f$ ) was lower than the critical level necessary to offset population losses $\left(f_{c}\right)$, the entire metapopulation went extinct, regardless of the level of stochastic disturbance of each local population. In contrast, when dispersal was asymmetrical, small stochastic perturbations to population abundances could grow transiently. When such perturbations were provided continuously in the form of stochastic noise, large increases in both the long-term average and variance of the metapopulation were observed, even when the larval production rate was below the critical level required to sustain the metapopulation. As we discuss below, these results have great implications for our understanding of the sources of population persistence in dispersal metapopulations in general, and on sustainable management and conservation of marine populations in particular.

There is no doubt that all natural populations occupying a finite region of land or ocean face temporally variable conditions that can disrupt, cause regional extinctions, transient responses, or sometimes even stabilize population dynamics and species coexistence (Chesson 1994, Chesson et al. 2004, Byers \& Pringle 
2006, Snyder 2009). We show that the inclusion of a very low-amplitude white noise process, representative of variability in environmental conditions that may average out over longer time scales and therefore be disregarded as ecologically unimportant by empirical ecologists (Wootton et al. 2009), can have dramatic consequences on metapopulation persistence whenever the pattern of connectivity among local populations is not symmetrical. Indeed, the stochastic variability can rescue the regional metapopulation from extinction. This result suggests that observed persistent dynamics in spatially structured systems cannot simply be interpreted as viable demographic parameters under the assumption that stable solutions are insensitive to environmental variability. The persistence of a species may be sustained for hundreds of generations solely by the existence of sufficient dispersal variability. Over longer time scales, environmental conditions are expected to change as a result of global climate change, and much research is dedicated to predict the long-term consequences of such variation on population viability and distribution. Our results on the sensitivity to variation of the model parameters themselves, such as the exact details of connectivity or mortality, demonstrate that metapopulations inhabiting finite ranges with asymmetrical dispersal may also be more susceptible to climate change than those connected by symmetrical dispersal.

For the simple dispersal kernels employed in our idealized linear metapopulation, the physical mechanism allowing transient perturbation growth is relatively straightforward, and it is simple to identify the optimal location to perturb the metapopulation. But under most natural situations, even along relatively straight coastlines, dispersal kernels are spatially and temporally variable, generating complex patterns of connectivity along the coast (Cowen et al. 2000, 2006, Guizien et al. 2006, Aiken et al. 2007). However, the same methods of generalized stability theory presented here can equally be used to identify sensitivities of metapopulations defined by the spatially and temporally complex connectivity patterns that occur in nature. With the SVD analysis, it is straightforward to determine the potentially most sensitive populations with respect to dispersal at any chosen PLD time scale, even when connectivity is highly complex and time dependent. When models of dispersal exist that have been evaluated for specific species (e.g. Gilg \& Hilbish 2000, Cowen et al. 2006, Guizien et al. 2006), the analysis presented here can be of great relevance for the sustainable management and conservation of marine populations. For instance, this approach aids identification of the most sensitive sector of the coast that, if protected from harvesting, i.e. establishing an MPA or a zonal prohibition of extraction, would have the great- est impact on the dynamics and population levels of the entire metapopulation. Theoretical research on the impact of MPAs has shown that, for the MPA strategy to be effective in aiding conservation and management of over-exploited species, a comparatively large, and in many ways unfeasible fraction ( $>35 \%$ ) of the coastline must be set aside as no-take reserves (Botsford et al. 2001, Lockwood et al. 2002, Gerber et al. 2003). Most of this research has been based on the assumption of symmetrical, spatially homogeneous and timeinvariant dispersal of larvae (but see Kaplan 2006) over the heterogeneous adult population landscape created by the reserve network. Therefore, further research using the generalized stability theory outlined here should re-evaluate the size and configuration of reserves that can maximize fisheries yields and save a rare species from extinction. Besides allowing identification of the perturbations that create the largest response in the metapopulation, generalized stability analysis can allow quantification of the interdependence of populations across the metapopulation and the time scales involved. In the example of central Chile given above, the sensitivity information provided by the SVD analysis suggests that increased (decreased) protection afforded to the northerly populations would have a 4 -fold positive (negative) impact upon abundances in particular southern populations.

Metapopulation dynamics are determined by the complex interaction of a broad range of factors. In this study we have focused solely upon the role of the larval dispersal phase. Despite the simplicity of the model, substantial variability in local population abundances can be sustained by a low level of stochastic forcing alone, without the need for including more complicated mechanisms, but only when larval dispersal is asymmetrical. Similarly, variability in the model dynamics driven by connectivity fluctuations may cause the metapopulation to switch continually between stable and unstable states. Thus, a component of observed population variability may be due to natural variability related to the larval dispersal phase alone, and may be driven by random variations in abundance at upstream locations. This opens the possibility that observed large amplitude fluctuations in population abundances may have a non-deterministic source. Recent emphasis has been placed on the importance of self-recruitment for sustainability of benthic populations and marine reserve designs (Swearer et al. 2002, Warner \& Cowen 2002, Tapia \& Pineda 2007). This is indeed an important advancement in our thinking of marine populations from the primordial idea embraced by some ecologists that all larvae were exported and no self-recruitment was possible (Warner \& Cowen 2002). It should be borne in mind, however, that even under the most advective environment created in our 
idealized models, and certainly in the realistic scenario for the coast of Chile, some self recruitment always occurs. What is most important for the dynamics and sensitivity of the metapopulation is, however, the level of asymmetry in the dispersal kernel. Therefore, monitoring of marine reserves should include not only the abundances of adult stages within reserves, but also the abundance and recruitment within the region of long-distance dispersal of the species.

Acknowledgements. Comments by J. Shinen, A. Caro, and 2 anonymous reviewers helped us improve this presentation. This study was supported by the International Laboratory for Global Change, CSIC-PUC (LINC-Global). C.M.A. acknowledges postdoctoral support by the Andrew Mellon Foundation and Linc-Global. Additional funding while preparing this manuscript was provided by Fondap-Fondecyt 15001-0001 to the Center for Advanced Studies in Ecology and Biodiverity (CASEB), Fondecyt no. 1070335 to S.A.N. and Fondecyt no. 1100646 to C.M.A.

\section{LITERATURE CITED}

Aiken CM, Moore AM, Middleton JH (2003) Nonnormal perturbation growth in idealised island and headland wakes. Dyn Atmos Oceans 37:171-195

- Aiken CM, Navarrete SA, Castillo M, Castilla JC (2007) Along-shore larval dispersal kernels in a numerical ocean model of the central Chilean coast. Mar Ecol Prog Ser 339: $13-24$

Aiken CM, Castillo M, Navarrete SA (2008) A simulation of the Chilean Coastal Current and associated topographic upwelling near Valparaíso, Chile. Cont Shelf Res 28: 2371-2381

> Anderson KE, Nisbet RM, McCauley E (2008) Transient responses to spatial perturbations in advective systems. Bull Math Biol 70:1480-1502

Botsford LW, Castilla JC, Peterson CH (1997) The management of fisheries and marine ecosystems. Science 277: 509-515

Botsford LW, Hastings A, Gaines MS (2001) Dependence of sustainability on the configuration of marine reserves and larval dispersal distance. Ecol Lett 4:144-150

> Byers JE, Pringle JM (2006) Going against the flow: retention, range limits and invasions in advective environments. Mar Ecol Prog Ser 313:27-41

Chesson P (1994) Multispecies competition in variable environments. Theor Popul Biol 45:227-276

> Chesson P (2003) Quantifying and testing coexistence mechanisms arising from recruitment fluctuations. Theor Popul Biol 64:345-357

> Chesson P, Lee C (2005) Families of discrete kernels for modeling dispersal. Theor Popul Biol 67:241-256

Chesson P, Gebauer RLE, Schwinning S, Huntly N and others (2004) Resource pulses, species interactions, and diversity maintenance in arid and semi-arid environments. Oecologia 141:236-253

Cowen R, Lwiza K, Sponaugle S, Paris CB, Olson DB (2000) Connectivity of marine populations: open or closed? Science 287:857-859

Cowen R, Paris CB, Srinivasan A (2006) Scaling of connectivity in marine populations. Science 311:522-527

Edwards KP, Hare JA, Werner FE, Seim H (2007) Using 2- dimensional dispersal kernels to identify the dominant influences on larval dispersal on continental shelves. Mar Ecol Prog Ser 352:77-87

Farrell BF, Ioannou PJ (1996) Generalized stability theory. Part I: Autonomous operators. J Atmos Sci 53:2025-2040

> Gaines SD, Bertness MD (1992) Dispersal of juveniles and variable recruitment in sessile marine species. Nature 360: 579-580

Gaines SD, Gaylord B, Gerber LR, Hastings A, Kinlan BP (2007) Connecting places: the ecological consequences of dispersal in the sea. Oceanography (Wash DC) 20:90-99

Gardiner CW (1985) Handbook of stochastic methods. Springer-Verlag, New York, NY

> Gerber LR, Botsford LW, Hastings A, Possingham HP, Gaines SD, Palumbi SR, Andelman S (2003) Population models for marine reserve design: a retrospective and prospective synthesis. Ecol Appl 13:47-64

Gilg MR, Hilbish TJ (2000) The relationship between allele frequency and tidal height in a mussel hybrid zone: a test of the differential settlement hypothesis. Mar Biol 137: 371-378

Golub GH, Van Loan CE (1989) Matrix computations. The Johns Hopkins University Press, Baltimore, MD

Guizien K, Brochier T, Duchene JC, Koh BS, Marsaleix P (2006) Dispersal of Owenia fusiformis larvae by wind-driven currents: turbulence, swimming behaviour and mortality in a three-dimensional stochastic model. Mar Ecol Prog Ser 311:47-66

> Hanski I, Gilpin M (1991) Metapopulation dynamics: brief history and conceptual domain. Biol J Linn Soc 42:3-16

Hanski I, Simberloff D (1997) The metapopulation approach, its history, conceptual domain, and application to conservation. In: Hanski I, Gilpin M (eds) Metapopulation biology: ecology, genetics and evolution. Academic Press, San Diego, CA, p 5-26

- Hastings A (2004) Transients: the key to long-term ecological understanding? Trends Ecol Evol 19:39-45

> Hastings A, Botsford LW (1999) Equivalence in yield from marine reserves and traditional fisheries management. Science 284:1537-1538

- Hastings A, Botsford LW (2006) Persistence of spatial populations depends on returning home. Proc Natl Acad Sci USA 103:6067-6072

Hastings A, Higgins K (1994) Persistence of transients in spatially structured ecological models. Science 263: 1133-1136

Jackson JBC, Kirby MX, Berger WH, Bjorndal KA and others (2001) Historical overfishing and the recent collapse of coastal ecosystems. Science 293:629-638

Kaplan D (2006) Alongshore advection and marine reserves: consequences for modeling and management. Mar Ecol Prog Ser 309:11-24

Kaplan DM, Botsford LW (2005) Effects of variability in spacing of coastal marine reserves on fisheries yield and sustainability. Can J Fish Aquat Sci 62:905-912

Katul GG, Porporato A, Nathan R, Siqueira M and others (2005) Mechanistic analytical models for long-distance seed dispersal by wind. Am Nat 166:368-381

Largier JL (2003) Considerations in estimating larval dispersal distances from oceanographic data. Ecol Appl 13: 71-89

Levins R (1969) Some demographic and genetic consequences of environmental heterogeneity for biological control. Bull Entomol Soc Am 15:237-240

Lewontin RC (1969) The bases of conflict in biological explanation. J Hist Biol 2:35-45

Lipcius RN, Stockhausen WT, Eggleston DB (2001) Marine 
reserves for Caribbean spiny lobster: empirical evaluation and theoretical metapopulation recruitment dynamics. Mar Freshw Res 52:1589-1598

Lockwood DR, Hastings A, Botsford LW (2002) The effect of dispersal patterns on marine reserves: Does the tail wag the dog? Theor Popul Biol 61:297-309

Lutscher F, Nisbet RM, Pachepsky E (2010) Population persistence in the face of advection. Theor Ecol 3:271-184

Marquet PA, Velasco-Hernández JX (1997) A source-sink patch occupancy metapopulation model. Rev Chil Hist Nat 70:371-380

Neubert MG, Caswell H (1997) Alternatives to resilience for measuring the responses of ecological systems to perturbations. Ecology 78:653-665

Neubert MG, Caswell H, Murray JD (2002) Transient dynamics and pattern formation: reactivity is necessary for Turing instabilities. Math Biosci 175:1-11

O'Connor MI, Bruno JF, Gaines SD, Halpern BS, Lester SE, Kinlan BP, Weiss JM (2007) Temperature control of larval dispersal and the implications for marine ecology, evolution, and conservation. Proc Natl Acad Sci USA 104: 1266-1271

Pachepsky E, Lutscher F, Nisbet RM, Lewis MA (2005) Persistence, spread and the drift paradox. Theor Popul Biol 67: 61-73

Palumbi SR (1994) Genetic divergence, reproductive isolation, and marine speciation. Annu Rev Ecol Syst 25: $547-572$

Paris CB, Chérubin LM, Cowen RK (2007) Surfing, spinning, or diving from reef to reef: effects on population connectivity. Mar Ecol Prog Ser 347:285-300

Pulliam HR (2000) On the relationship between niche and distribution. Ecol Lett 3:349-361

Roughgarden J (2006) Foreword. In: Kritzer J, Sale PF (eds) Marine metapopulations. Elsevier Academic Press, San Diego, CA, p xii-xx

Roughgarden J, Iwasa Y, Baxter C (1985) Demographic theory for an open marine population with space-limited recruitment. Ecology 66:54-67
Sale PF, Hanski I, Kritzer J (2006) The merging of metapopulation theory and marine ecology: establishing the historical context. In: Kritzer J, Sale PF (eds) Marine metapopulations. Elsevier Academic Press, San Diego, CA, p 3-29

Siegel DA, Mitarai S, Costello CJ, Gaines SD, Kendall BE, Warner R, Winters KB (2008) The stochastic nature of larval connectivity among nearshore marine populations. Proc Natl Acad Sci USA 105:8974-8979

Snyder RE (2009) Transient dynamics in altered disturbance regimes: recovery may start quickly, then slow. Theor Ecol 2:79-87

Snyder RE, Chesson P (2003) Local dispersal can facilitate coexistence in the presence of permanent spatial heterogeneity. Ecol Lett 6:301-309

Speirs DC, Gurney WSC (2001) Population persistence in rivers and estuaries. Ecology 82:1219-1237

Swearer SE, Shima JS, Hellberg ME, Thorrold SR and others (2002) Evidence of self-recruitment in demersal marine populations. Bull Mar Sci 70:251-271

Tapia FJ, Pineda J (2007) Stage-specific distribution of barnacle larvae in nearshore waters: potential for limited dispersal and high mortality rates. Mar Ecol Prog Ser 342: $177-190$

Taylor AD (1990) Metapopulation, dispersal, and predatorprey dynamics: an overview. Ecology 71:429-433

Trefethen LN, Trefethen AE, Reddy SC, Driscoll TA (1993) Hydrodynamic stability without eigenvalues. Science 261: 578-584

Warner RR, Cowen R (2002) Local retention of production in marine populations: evidence, mechanisms, and consequences. Bull Mar Sci 70:245-249

Wieters EA, Gaines SD, Navarrete SA, Blanchette C, Menge BA (2008) Scales of dispersal and the biogeography of marine predator-prey interactions. Am Nat 171:405-417

Wootton JT, Cusson M, Navarrete SA, Petraitis PS (2009) Disruptions, succession and stochasticity. In: Wahl M (ed) Marine hard bottom communities. Springer-Verlag, Berlin, p 201-212

Appendix 1. Singular value decomposition

In general, non-normality may be diagnosed by the fact that $\mathbf{A}$ does not commute with the adjoint operator $\mathbf{A}^{+}$, that is $\mathbf{A}^{+} \mathbf{A} \neq \mathbf{A A}^{+}$(Farrell \& Ioannou 1996). While Lyapunov or eigenanalysis of $\mathbf{A}$ predicts the asymptotic behavior of the system, the dynamics over the transient time scale $t$ may be determined through the singular value decomposition (SVD; Golub \& Van Loan 1989) of the dynamical system's 'propagator' $\mathbf{R}(t)=\mathrm{e}^{\mathbf{A} t}$. The propagator represents the action of the tangent linear model (TLM) over time $t_{i}$ that is, it is the function that gives the new set of abundances based on their past values (Farrell \& Ioannou 1996). An SVD corresponds to performing eigenanalysis of the symmetrical operator $\mathbf{R}^{+} \mathbf{R}$. When the propagator is represented in matrix form, its adjoint is simply the Hermitian transpose. The SVD yields orthogonal bases in the domain and range space:

$$
\mathbf{R}(t)=\mathbf{U} \Sigma \mathbf{V}^{+}
$$

where each column of $\mathbf{V}$ represents an initial condition that is mapped to a final state given by the corresponding row of U. The amplification factor of the mapping is given by the corresponding element of the diagonal matrix $\boldsymbol{\Sigma}$, referred to as the singular values. Thus, the possibility for perturbations to grow over transient time scales can be determined through performance of an SVD on the propagator $\mathbf{R}(t)$ for a range of times $t$.
Editorial responsibility: Romuald Lipcius, Gloucester Point, Virginia, USA
Submitted: June 2, 2010; Accepted: February 9, 2011

Proofs received from author(s): April 21, 2011 